\title{
Validation of Methods for Ranking Fuzzy Numbers in Decision Making
}

\author{
Alexander Gegov* and Ahmad Syafadhli Abu Bakar \\ School of Computing, University of Portsmouth, Portsmouth PO1 3HE, United Kingdom \\ *Corresponding author. Address: School of Computing, University of Portsmouth, Buckingham Building, \\ Portsmouth PO1 3HE, United Kingdom. \\ Tel: +44 (0)239284 6412; Fax: +44(0)2392846411 \\ Email address: alexander.gegov@port.ac.uk
}

\begin{abstract}
The concept of ranking fuzzy numbers has attracted significant attention recently due to its successful use in decision making problems. This concept allows decision makers to appropriately exercise their subjective judgement under situations that are vague, imprecise, ambiguous and uncertain in nature. The literature on ranking fuzzy numbers describes the validation of ranking methods as the most important aspect of the application of these methods. This is due to the fact that the validation confirms the suitability of the associated methods for ranking fuzzy numbers and decision making purposes. In this paper, a comprehensive review on validation techniques for methods of ranking fuzzy numbers is presented. These techniques are associated with properties of ranking fuzzy quantities as well as consistency and efficiency evaluation of ranking operations. The techniques are described in detail and discussed in the context of many established and more recent works in the field.
\end{abstract}

Keywords: Ranking, fuzzy numbers, type -1 fuzzy numbers, type -2 fuzzy numbers, $Z$ - numbers, ranking properties, consistency, efficiency.

\section{Introduction}

Ranking fuzzy numbers is used in fuzzy sets as a concept that determines which fuzzy number is greater when two or more fuzzy numbers are compared. A definition by (Collan, 2009) refers ranking fuzzy numbers as a process of comparing and organising fuzzy numbers in a specific order. The definition indicates that each fuzzy number under consideration is assigned a value whereby this value is used as a comparison measure with other fuzzy numbers. Then, values obtained from each fuzzy number under consideration are compared accordingly. Even though the process for ranking fuzzy numbers mentioned looks simple, to naturally determine the correct ordering for each fuzzy number under consideration is not easy as fuzzy numbers are represented by possibility distribution (Zimmermann, 2000; Kumar et al., 2010). Therefore, finding an appropriate ranking method is important as fuzzy numbers are often used in various decision making situations such as risk analysis by Chen et al., (2012), supply chain management (Wu et al., 2013), fuzzy portfolio (Bermudez et al., 2012), selection of construction project (Ebrahimnejad et al., 2012) and decision making for water resources (Morais\& Almeida, 2012).

In the literature on fuzzy sets, there are three main types of fuzzy numbers. These numbers are also introduced here - namely, type - 1 fuzzy numbers (Zadeh, 1965), type - 2 fuzzy numbers (Zadeh, 1975) and Z - numbers (Zadeh, 2011). Type - 1 fuzzy number is the first type of fuzzy number that can deal with imprecise numerical quantities and subjective preference of decision makers (Deng, 2013). Type -2 fuzzy number is an extension of type - 1 fuzzy number that takes into account modelling perceptions. This extension is made because uncertainty in terms of natural language, which is represented by type -1 fuzzy number, is insufficient to model perception (Dereli et al., 2011). In addition, the imprecision level about a situation is increased during transition from numbers to words of natural language and finally to perceptions (John \&Coupland, 2009). This strengthens the argument against the less capable with respect to model perception type -1 fuzzy number. $Z$ - number is another extension of type -1 fuzzy number that can deal with decision making problems where reliability of information very important (Kang et al., 2012a, b). According to (Zadeh, 2011), $Z$ - number is considered as a generalisation of numbers, intervals, fuzzy numbers and 
random numbers because it is ranked with the highest level of generality - level 3 compared to numbers (ground level 1), intervals (level 1), fuzzy numbers (level 2) and random numbers (level 2). In this respect, if the level of generality of a number is higher than the one for other numbers, then the capability of this number to construct a realistic model of a real world system is greater (Zadeh, 2011). Therefore, $\mathrm{Z}$ - numbers are justified as having a better representation capability compared to type -1 and type -2 fuzzy numbers.

There are a lot of methods in the literature on fuzzy sets for ranking type - 1 fuzzy numbers such as ranking methods based on area (Wang et al., 2005), (Kumar et al., 2010), (Chen \& Sanguatsan, 2011), (Thorani et al., 2013), ranking methods using centroid approach (Cheng, 1998), (Chu \& Tsao, 2002), (Wang \& Yang, 2006), (Chen \& Chen, 2009), (Wang \& Lee, 2009), (Bakar et al., 2010) and ranking method based on distance (Yao \& Wu, 2000), (Asady \& Zendehnam, 2007), (Asady, 2009), (Asady \& Abbasbandy, 2009), (Rao \& Shankar, 2013), (Wang et al., 2013), (Bakar \& Gegov, 2014). However, there seem to be only a few ranking methods for type - 2 fuzzy numbers (Mitchel, 2006), (Wu \& Mendel, 2009) and ranking $Z$ - numbers (Mohamad et al., 2014), (Bakar \& Gegov, 2015).

In order to determine the suitability of a method for ranking fuzzy numbers in decision making applications, each ranking method is evaluated using both theoretical and empirical validation that is established in the literature on ranking fuzzy numbers. The validation is based on reasonable theoretical properties for ranking fuzzy quantities of type -1 and type -2 fuzzy numbers (Wang \& Kerre, 2001; 2002), numerical cases of type -1 fuzzy numbers (Chen $\&$ Chen, 2009), numerical cases of type -2 fuzzy numbers (Wu \& Mendel, 2009) and numerical cases of $Z$ - numbers (Bakar \& Gegov, 2015). In this context, the paper reviews all above mentioned aspects of validation and shows detailed comparison among established ranking methods based on the type of validation used.

The remainder of the paper is organised as follows: Section 2 discusses theoretical preliminaries for this study, Section 3 provides details on validation for ranking methods and Section 4 presents a conclusion.

\section{Theoretical preliminaries}

Trapezoidal fuzzy number is illustrated in this section as a special case of triangular fuzzy number (Chen \& Chen, 2009). Non - linear fuzzy numbers are not considered in this section as linear fuzzy numbers are often used in many decision making situations and discussions on utilisation of linear fuzzy numbers are frequently discussed in the literature as opposed to non - linear ones. Furthermore, non - linear fuzzy numbers are too complex to handle and they are often transformed into linear type for convenience (Chen \& Linkens, 2004).

\section{A. Type - 1 Fuzzy Numbers}

In accordance with (Chen \& Chen, 2009), the type -1 fuzzy numbers shown in Figure 1 can be represented by the following membership function

$$
\mu_{A}(x)=\left(a_{1}, a_{2}, a_{3}, a_{4}\right)=\left\{\begin{array}{ccc}
\frac{x-a_{1}}{a_{2}-a_{1}} & \text { if } & a_{1} \leq x \leq a_{2} \\
1 & \text { if } & a_{2} \leq x \leq a_{3} \\
\frac{a_{4}-x}{a_{4}-a_{3}} & \text { if } & a_{3} \leq x \leq a_{4} \\
0 & \text { otherwise }
\end{array}\right.
$$




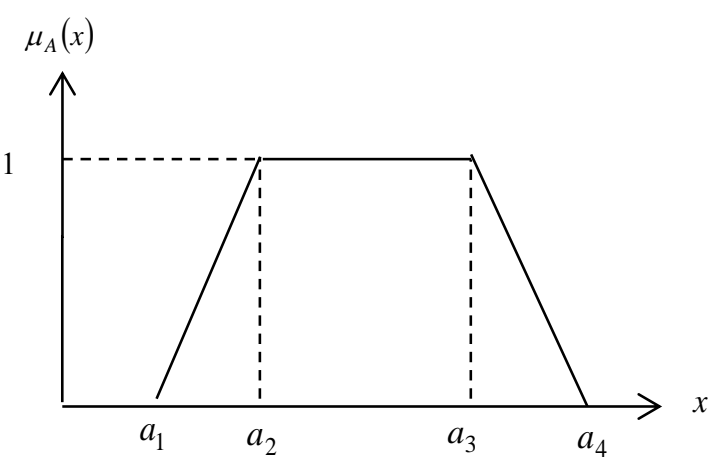

Figure 1: Type - 1 Fuzzy Number

\section{B. Type - 2 Fuzzy Numbers}

According to (Wallsten \& Budescu, 1995), a type - 2 fuzzy number can be viewed as a group of type - 1 fuzzy numbers. The type -2 fuzzy number shown in Figure 2 can be represented by the following membership functions

$$
\begin{gathered}
\mu_{A_{U}}(x)=\left(a_{U 1}, a_{U 2}, a_{U 3}, a_{U 4}\right)=\left\{\begin{array}{cll}
\frac{x-a_{U 1}}{a_{U 2}-a_{U 1}} & \text { if } & a_{U 1} \leq x \leq a_{U 2} \\
w_{A_{U}} & \text { if } & a_{U 2} \leq x \leq a_{U 3} \\
\frac{a_{U 4}-x}{a_{U 4}-a_{U 3}} & \text { if } & a_{U 3} \leq x \leq a_{U 4} \\
0 & \text { otherwise }
\end{array}\right. \\
\mu_{A_{L}}(x)=\left(a_{L 1}, a_{L 2}, a_{L 3}, a_{L 4}\right)=\left\{\begin{array}{ccc}
\frac{x-a_{L 1}}{a_{L 2}-a_{L 1}} & \text { if } & a_{L 1} \leq x \leq a_{L 2} \\
\frac{w_{A_{L}}}{a_{L 4}-x} & \text { if } & a_{L 2} \leq x \leq a_{L 3} \\
\frac{a_{L 4}-a_{L 3}}{0} & \text { if } & a_{L 3} \leq x \leq a_{L 4} \\
0 & \text { otherwise }
\end{array}\right.
\end{gathered}
$$

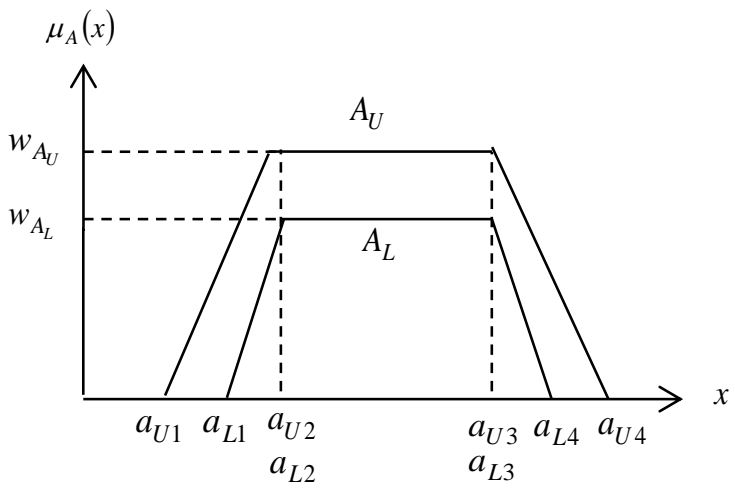

Figure 2: Type - 2 Fuzzy Number 
C. $Z$ - numbers

According to (Zadeh, 2011), a $Z$ - number is an ordered pair of fuzzy number denoted as $Z=(\bar{A}, \bar{B})$. The first component $\bar{A}$ is a restriction component in the form of a real - valued uncertainty on $X$ whereas the second component $\bar{B}$ is a measure of reliability for $\bar{A}$. In Accordance with (Kang et al., 2012b, the $Z$ - number shown in Figure 3 can be represented by the following membership functions

$$
\begin{gathered}
\mu_{\bar{A}}(x)=\left(a_{1}, a_{2}, a_{3}, a_{4}\right)=\left\{\begin{array}{ccc}
\frac{x-a_{1}}{a_{2}-a_{1}} & \text { if } & a_{1} \leq x \leq a_{2} \\
1 & \text { if } & a_{2} \leq x \leq a_{3} \\
\frac{a_{4}-x}{a_{4}-a_{3}} & \text { if } & a_{3} \leq x \leq a_{4} \\
0 & \text { otherwise }
\end{array}\right. \\
\mu_{\bar{B}}(x)=\left(b_{1}, b_{2}, b_{3}, b_{4}\right)=\left\{\begin{array}{ccc}
\frac{x-b_{1}}{b_{2}-b_{1}} & \text { if } & b_{1} \leq x \leq b_{2} \\
1 & \text { if } & b_{2} \leq x \leq b_{3} \\
\frac{b_{4}-x}{b_{4}-b_{3}} & \text { if } & b_{3} \leq x \leq b_{4} \\
0 & \text { otherwise }
\end{array}\right.
\end{gathered}
$$

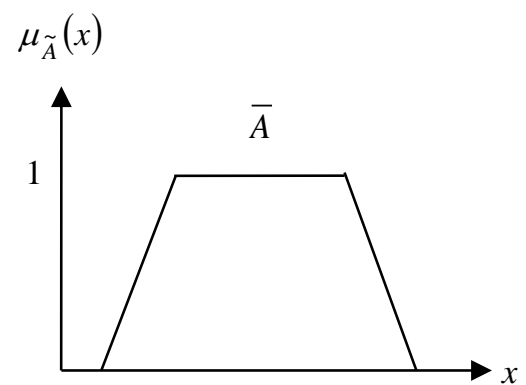

$\bar{A}=\left[a_{1}, a_{2}, a_{3}, a_{4} ; 1\right]$
$\mu_{\tilde{B}}(x)$

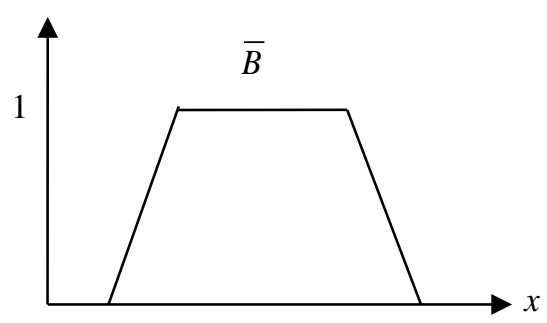

$\bar{B}=\left[b_{1}, b_{2}, b_{3}, b_{4} ; 1\right]$

Figure 3: A $Z$ - number, $Z=(\bar{A}, \bar{B})$

\section{Validation Techniques for Ranking Methods}

According to the literature on ranking fuzzy numbers, validation techniques for ranking methods can be classified in two groups - theoretical and empirical validation. In research works by (Chen \& Chen, 2009), (Dat et al., 2012), (Brunelli \& Mezei, 2013) and (Bakar \& Gegov, 2015), theoretical validation is broadly concerned with fulfilment of generic properties by ranking methods whereas the empirical validation concentrates on the consistency and efficiency of a ranking method. Therefore, without loss of generality, discussion on both theoretical and empirical validation for ranking methods is presented below.

\section{A. Theoretical Validation}

According to Brunelli \& Mezei (2013), this validation is an axiomatic based - research as it concerns with a broad scope of ranking fuzzy numbers where ranking methods are validated based on reasonable properties for ranking fuzzy 
quantities. Fuzzy quantities defined by Wang \& Kerre (2001) are in principle more generic than fuzzy numbers, but they are not often used in the literature of fuzzy sets. Since, fuzzy numbers are subsets of fuzzy quantities, hence any properties that are related to the latter are also applicable to the former. In the literature of fuzzy sets, reasonable properties for ranking fuzzy quantities are presented by Wang \& Kerre $(2001 ; 2002)$ where these properties are purposely developed for type -1 and type - 2 fuzzy numbers. Wu \& Mendel (2009), Kumar et al. (2010), Asady (2010) and $\mathrm{Yu}$ et al. (2013) are among the recently established ranking methods that utilise these properties in validating their methods. Therefore, based on Wang \& Kerre $(2001,2002)$, reasonable properties for ranking fuzzy quantities which is fuzzy numbers are as follows.

Let $\tilde{A}_{1}$ and $\tilde{A}_{2}$ be two fuzzy numbers such that $\tilde{A}_{1}$ and $\tilde{A}_{2}$ are any types of fuzzy numbers.

Property 1: If $\tilde{A}_{1} \geqslant \tilde{A}_{2}$ and $\tilde{A}_{2} \geqslant \tilde{A}_{1}$, then $\tilde{A}_{1} \approx \tilde{A}_{2}$

Property 2: If $\tilde{A}_{1} \geqslant \tilde{A}_{2}$ and $\tilde{A}_{2} \geqslant \tilde{A}_{3}$, then $\tilde{A}_{1} \geqslant \tilde{A}_{3}$

Property 3: If $\tilde{A}_{1} \cap \tilde{A}_{2}=\emptyset$ and $\tilde{A}_{1}$ is on right side of $\tilde{A}_{2}$, then $\tilde{A}_{1} \geqslant \tilde{A}_{2}$

Property 4: Ordering of $\tilde{A}_{1}$ and $\tilde{A}_{2}$ is not affected by other fuzzy numbers under comparison.

Property 5: If $\tilde{A}_{1} \geqslant \tilde{A}_{2}$, then $\tilde{A}_{1}+\tilde{C} \geqslant \tilde{A}_{2}+\tilde{C}$, where $\tilde{C}$ is constant in fuzzy number.

Property 6: If $\tilde{A}_{1} \geqslant \tilde{A}_{2}$, then $\tilde{A}_{1} \tilde{C} \geqslant \tilde{A}_{2} \tilde{C}$, where $\tilde{C}$ is constant in fuzzy number.

If a ranking method fulfils all the aforementioned ranking properties suggested by Wang \& Kerre $(2001 ; 2002)$, then the method is considered as an effective ranking method theoretically. Table 1 illustrates the applicability of the properties of ranking fuzzy quantities towards fuzzy numbers.

Table 1: Applicability of the properties of ranking fuzzy quantities towards fuzzy numbers.

\begin{tabular}{lc}
\hline Fuzzy Numbers & Properties Applicability \\
\hline Type -1 & Yes \\
\hline Type -2 & Yes \\
\hline Type -2 after reduction into Type -1 & Yes \\
\hline$Z-$ numbers & No \\
\hline$Z-$ numbers after reduction into Type -1 & Yes \\
\hline
\end{tabular}

Although, the aforementioned properties are not developed for $\mathrm{Z}$ - numbers in the first place, as $\mathrm{Z}$ - numbers are new in the literature of fuzzy sets (Zadeh, 2011), they are all applicable whenever Z - numbers are reduced into type - 1 fuzzy numbers (Kang et al., 2012). Table 2 illustrates comparison in terms of the properties fulfilment by established ranking methods. 
Table 2: Comparison on properties fulfilments by ranking methods.

\begin{tabular}{|c|c|c|c|c|c|c|c|}
\hline \multirow{2}{*}{ Ranking Method } & \multicolumn{6}{|c|}{ Property } & \multirow{2}{*}{ Properties fulfilment } \\
\hline & 1 & 2 & 3 & 4 & 5 & $\mathbf{6}$ & \\
\hline 1 - Cheng (1998) & $\mathrm{Y}$ & $\mathrm{N}$ & $\mathrm{N}$ & $\mathrm{Y}$ & $\mathrm{N}$ & $\mathrm{Y}$ & $3 / 6$ \\
\hline 2 - Wu \& Mendel (2009) & $\mathrm{Y}$ & Y & $\mathrm{Y}$ & $\mathrm{Y}$ & Y & Y & $6 / 6$ \\
\hline 1 - Kumar et al. (2010) & $\mathrm{Y}$ & $\mathrm{N}$ & $\mathrm{N}$ & $\mathrm{Y}$ & $\mathrm{N}$ & $\mathrm{Y}$ & $3 / 6$ \\
\hline 1 - Dat et al. (2012) & $\mathrm{Y}$ & $\mathrm{Y}$ & $\mathrm{Y}$ & Y & $\mathrm{Y}$ & Y & $6 / 6$ \\
\hline $1-$ Yu et al. (2013) for $\alpha=0$ & $\mathrm{Y}$ & $\mathrm{N}$ & $\mathrm{Y}$ & $\mathrm{Y}$ & $\mathrm{Y}$ & $\mathrm{Y}$ & $5 / 6$ \\
\hline 1 - Yu et al. (2013) for $\alpha=0.5$ & $\mathrm{Y}$ & $\mathrm{N}$ & $\mathrm{Y}$ & $\mathrm{Y}$ & $\mathrm{Y}$ & $\mathrm{Y}$ & $5 / 6$ \\
\hline $1-$ Yu et al. (2013) for $\alpha=1$ & $\mathrm{Y}$ & $\mathrm{N}$ & $\mathrm{Y}$ & $\mathrm{Y}$ & $\mathrm{Y}$ & $\mathrm{Y}$ & $5 / 6$ \\
\hline $1-$ Zhang et al. (2014) for $\alpha=0$ & $\mathrm{Y}$ & $\mathrm{N}$ & $\mathrm{Y}$ & $\mathrm{Y}$ & $\mathrm{Y}$ & $\mathrm{Y}$ & $5 / 6$ \\
\hline $1-$ Zhang et al. (2014) for $\alpha=0.5$ & $\mathrm{Y}$ & $\mathrm{N}$ & $\mathrm{Y}$ & $\mathrm{Y}$ & $\mathrm{Y}$ & Y & $5 / 6$ \\
\hline 1 - Zhang et al. (2014) for $\alpha=1$ & $\mathrm{Y}$ & $\mathrm{N}$ & $\mathrm{Y}$ & $\mathrm{Y}$ & $\mathrm{Y}$ & Y & $5 / 6$ \\
\hline 1 - Bakar \& Gegov (2014) & $\mathrm{Y}$ & $\mathrm{Y}$ & $\mathrm{Y}$ & $\mathrm{Y}$ & $\mathrm{Y}$ & $\mathrm{Y}$ & $6 / 6$ \\
\hline Z - Bakar \& Gegov (2015) & $\mathrm{Y}$ & $\mathrm{Y}$ & $\mathrm{Y}$ & $\mathrm{Y}$ & $\mathrm{Y}$ & $\mathrm{Y}$ & $6 / 6$ \\
\hline
\end{tabular}

' $\mathrm{Y}$ ' denotes that the ranking method satisfies the property

' $\mathrm{N}$ ' denotes that the ranking method dissatisfies the property

' 1 ' denotes that the ranking method is developed for ranking type -1 fuzzy numbers

' 2 ' denotes that the ranking method is developed for ranking type -2 fuzzy numbers

' $\mathrm{Z}$ ' denotes that the ranking method is developed for ranking $\mathrm{Z}$ - number.

\section{B. Empirical Validation}

For this validation, discussions are made in accordance to case studies found in the literature of fuzzy sets. Among the case studies found are risk analysis under uncertainty (Chen \& Chen, 2009), fuzzy programming in textile industry (Elamvazuthi et al., 2009), fuzzy approach in torque - sensorless control of DC motor (Liem et al., 2015), inspection planning in manufacturing problem (Mousavi et al., 2015) and uncertain stochastic nonlinear systems with input saturation (Sui et al., 2015). Based on these case studies, Cheng (1998), Wang et al. (2005), Asady (2009), Chen \& Chen (2007, 2009), Dat et al. (2012), Yu et al. (2013) and Bakar \& Gegov (2014; 2015) suggest several numerical examples that generically represent all of the aforementioned case studies. All numerical examples presented in the literature are explained and illustrated as follows.

\section{Trivial Case}

Trivial case involves cases of fuzzy numbers which are simple and easy to differentiate whereby ranking order of this category is determined by observing the nature of fuzzy numbers under consideration. This case is intentionally considered in this study to demonstrate the capability of ranking method in ranking simple fuzzy numbers cases before being applied to more complex fuzzy numbers cases. Figure 4 and 5 illustrate two generic fuzzy numbers examples of Trivial Case.

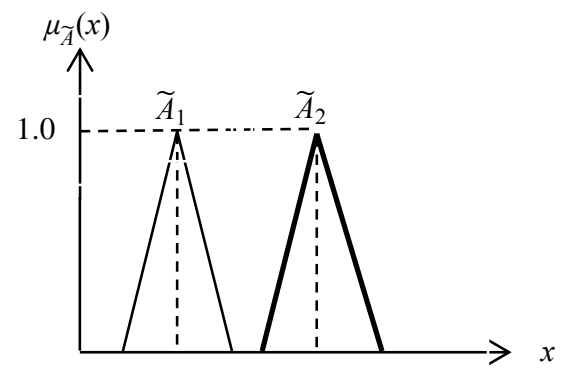

Figure 4: Trivial Case 1

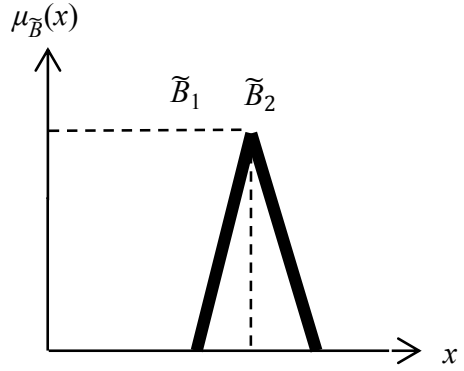

Figure 5: Trivial Case ? 


\section{Embedded Case}

Embedded case involves sets of fuzzy numbers which are fully overlapped with one to another. Not all ranking methods are capable to deal with embedded fuzzy numbers cases appropriately as many ranking methods neglect this case in their studies such as Lee \& Li (1988) and Cross \& Setnes (1998). Figure 6, 7 and 8 illustrate three generic fuzzy numbers examples of Embedded Case.

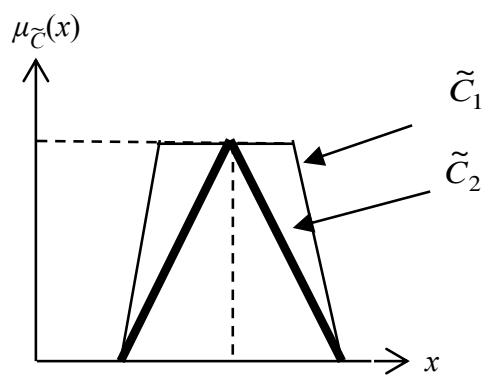

Figure 6: Embedded Case 1

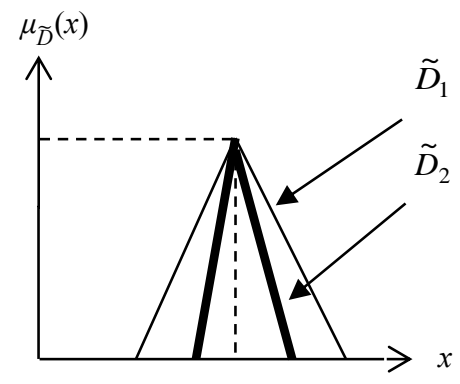

Figure 7: Embedded Case 2

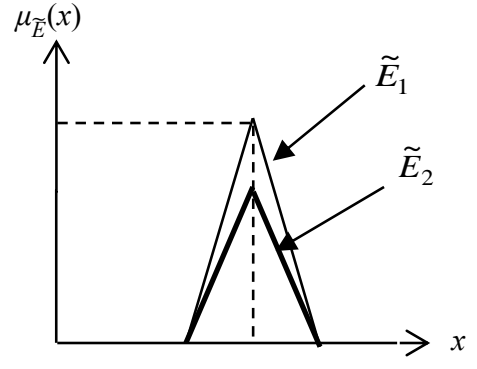

Figure 8: Embedded Case 3

\section{Overlapping Case}

Overlapping case category is among the important cases in ranking fuzzy numbers area of research. If embedded fuzzy numbers cases are fuzzy numbers which are fully overlapped with each other, this category considers fuzzy numbers that are partially overlapped from one to another. Figure 9 and 10 illustrate two generic fuzzy numbers examples of Overlapping Case.

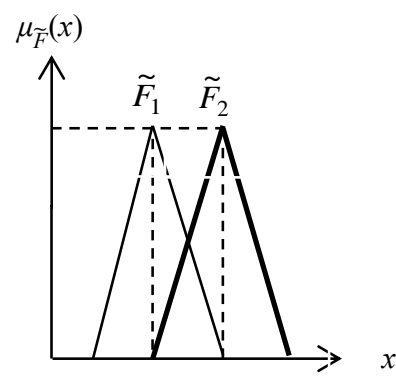

Figure 9: Overlapping Cásé 1

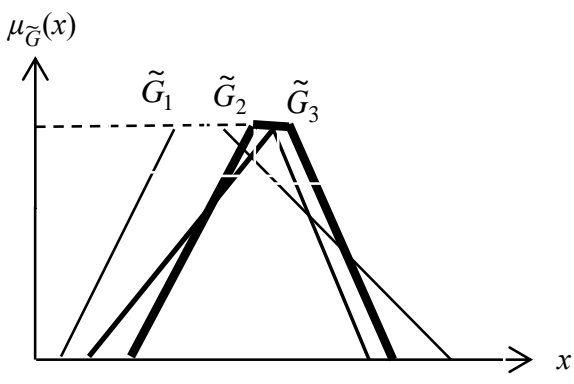

Figure 10: Overlapping Case 2

\section{Non-overlapping Case}

Non - overlapping case category involves cases of fuzzy numbers that are separated from each other. The cases include opposite cases from overlapping fuzzy numbers category. Figure 11 and 12 illustrate two generic fuzzy numbers examples of Non - Overlapping Case. 


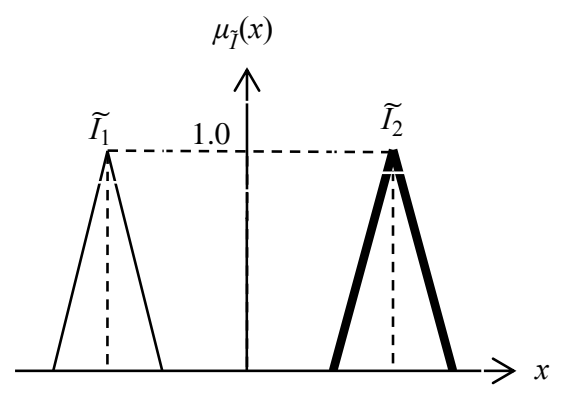

Figure 11: Non - Overlapping Case 1

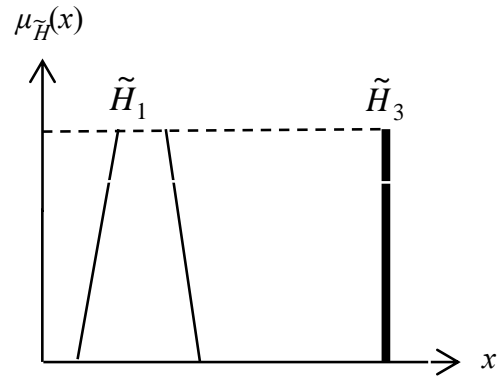

Figure 12: Non - Overlapping Case 2

There are two types of empirical validations are reviewed here namely consistency evaluation and efficiency evaluation where their descriptions are as follows.

\section{Consistency Evaluation}

Consistency is defined in ranking fuzzy numbers area of research as the capability of a ranking method to producing correct ranking order for any cases of fuzzy numbers such that ranking results obtained are consistent with ranking order obtained by human intuition. If a method ranks correctly fuzzy numbers such that the ranking results are consistent with human intuition, then the ranking result is considered as consistent. Otherwise ranking result obtained is classified as inconsistent. As mentioned earlier in the introduction, type -2 fuzzy numbers and $Z$ numbers are extension of type -1 fuzzy numbers because both type -2 fuzzy numbers and $\mathrm{Z}$ - numbers are usually reduced to type - 1 fuzzy numbers for easy computational works (Greenfield \& Chiclana, 2009; Kang et al., 2012) . Thus, the consistency evaluations for each fuzzy numbers are considered as empirically the same as they are all represented by possibility distribution (Kumar et al, 2010). Therefore, description on the consistency evaluation for each fuzzy number considered is as follows.

Let consider $\widetilde{A}$ and $\widetilde{B}$ as two fuzzy numbers to be ranked. Table 3 presents an ordering - based comparison in terms of consistency of a ranking method when ranking fuzzy numbers. It classifies all possible ranking order of fuzzy numbers whereby all these results are compared with result obtain by human intuition.

Table 3: Evaluation of Consistency for Two Fuzzy Numbers

\begin{tabular}{cc}
\hline \multicolumn{2}{c}{ Human intuition $=\tilde{A} \succ \tilde{B}$} \\
\hline Ranking order & Consistency \\
\hline$\tilde{A} \succ \tilde{B}$ & Consistent \\
$\tilde{B} \succ \tilde{A}$ & Inconsistent \\
$\tilde{A} \approx \tilde{B}$ & Inconsistent \\
\hline
\end{tabular}

While for three fuzzy numbers, let consider $\widetilde{A}, \widetilde{B}$ and $\widetilde{C}$ as three fuzzy numbers to be ranked where all possible ranking ordering of fuzzy numbers whereby all these results are compared with result obtain by human intuition are given in Table 4. 
Table 4: Evaluation of Consistency for Three Fuzzy Numbers

\begin{tabular}{cc}
\hline \multicolumn{2}{c}{ Human intuition $=\tilde{A} \succ \tilde{B} \succ \tilde{C}$} \\
\hline Ranking order & Consistency \\
\hline$\tilde{A} \succ \tilde{B} \succ \tilde{C}$ & Consistent \\
$\tilde{A} \succ \tilde{C} \succ \tilde{B}$ & Inconsistent \\
$\tilde{B} \succ \tilde{A} \succ \tilde{C}$ & Inconsistent \\
$\tilde{B} \succ \tilde{C} \succ \tilde{A}$ & Inconsistent \\
$\tilde{C} \succ \tilde{B} \succ \tilde{A}$ & Inconsistent \\
$\tilde{C} \succ \tilde{A} \succ \tilde{B}$ & Inconsistent \\
$\tilde{A} \approx \tilde{B} \succ \tilde{C}$ & Inconsistent \\
$\tilde{A} \succ \tilde{B} \approx \tilde{C}$ & Inconsistent \\
$\tilde{B} \succ \tilde{C} \approx \tilde{A}$ & Inconsistent \\
$\tilde{B} \approx \tilde{C} \succ \tilde{A}$ & Inconsistent \\
$\tilde{C} \approx \tilde{B} \succ \tilde{A}$ & Inconsistent \\
$\tilde{C} \succ \tilde{B} \approx \tilde{A}$ & Inconsistent \\
$\tilde{A} \approx \tilde{B} \approx \tilde{C}$ & Inconsistent \\
\hline
\end{tabular}

Based on Table 3 and 4, it is understandable that there is only one results that is consistent with ranking result obtained by human intuition and it is considered as a consistent ranking order. While, for other ranking orders, ranking results obtained are classified as inconsistent ranking orders. It is worth adding here that investigations on ranking fuzzy numbers are not only restricted for ranking two and three fuzzy numbers as Abbasbandy \& Asady (2006), Abbasbandy \& Hajjari (2009), Wang et al. (2009), Hadi \& Allame (2010) and Salahshour et al. (2011) consider more than three fuzzy numbers in their studies. Thus, Table 3 and 4 of two and three fuzzy numbers respectively are extendable so that they are fitted for other quantity of fuzzy numbers. In Table 5, comparisons of consistency evaluation among established ranking methods considered when ranking fuzzy numbers based on Figure 4 till Figure 12 are illustrated.

Table 5: Consistency comparison among ranking methods.

\begin{tabular}{|c|c|c|c|c|c|c|c|c|c|}
\hline \multirow{3}{*}{$\begin{array}{l}\text { Ranking } \\
\text { Method }\end{array}$} & \multicolumn{9}{|c|}{ Numerical Example } \\
\hline & \multicolumn{2}{|c|}{ Trivial } & \multicolumn{3}{|c|}{ Embedded } & \multicolumn{2}{|c|}{ Overlapping } & \multicolumn{2}{|c|}{ Non - Overlapping } \\
\hline & Case 1 & Case 2 & Case 1 & Case 2 & Case 3 & Case 1 & Case 2 & Case 1 & Case 2 \\
\hline 1 - Cheng (1998) & Yes & Yes & No & No & Yes & No & Yes & Yes & No \\
\hline 2-Wu \& Mendel (2009) & Yes & Yes & No & No & Yes & No & Yes & Yes & No \\
\hline 1 - Kumar et al. (2010) & No & Yes & No & No & No & No & Yes & No & No \\
\hline 1 - Dat et al. (2012) & Yes & Yes & No & No & Yes & Yes & Yes & Yes & Yes \\
\hline $1-$ Yu et al. (2013) for $\alpha=0$ & Yes & Yes & No & No & No & Yes & Yes & No & No \\
\hline $1-$ Yu et al. (2013) for $\alpha=0.5$ & Yes & Yes & No & No & No & Yes & Yes & No & No \\
\hline $1-$ Yu et al. (2013) for $\alpha=1$ & Yes & Yes & Yes & No & Yes & Yes & Yes & No & No \\
\hline $1-$ Zhang et al. (2014) for $\alpha=0$ & Yes & Yes & No & No & No & Yes & Yes & No & No \\
\hline $1-$ Zhang et al. (2014) for $\alpha=0.5$ & Yes & Yes & No & No & No & Yes & Yes & No & No \\
\hline 1 - Zhang et al. (2014) for $\alpha=1$ & Yes & Yes & No & No & No & Yes & Yes & No & No \\
\hline 1 - Bakar \& Gegov (2014) & Yes & Yes & Yes & Yes & Yes & Yes & Yes & Yes & Yes \\
\hline 2 - Bakar \& Gegov (2015) & Yes & Yes & Yes & Yes & Yes & Yes & Yes & Yes & Yes \\
\hline
\end{tabular}

' 1 ' denotes that the ranking method is developed for ranking type -1 fuzzy numbers

' 2 ' denotes that the ranking method is developed for ranking type -2 fuzzy numbers

' $Z$ ' denotes that the ranking method is developed for ranking $\mathrm{Z}$ - number. 
In Table 5, it is noticeable that not all ranking methods are capable of ranking specific fuzzy numbers cases with correct ranking order such that the results are consistent with human intuition. This is due to the fact that every ranking methods considered have their own advantages and disadvantages.

\section{Efficiency Evaluation}

According to Allahviranloo et al. (2013), Fries (2014) and Jahantigh \& Hajighasemi (2014), efficiency of a ranking method is often determined in accordance to its computational complexity when ranking fuzzy numbers. In the literature of fuzzy sets, two kinds of ranking method are found namely direct ranking and pairwise ranking. Direct ranking refers to the capability of a method to ranking any quantity of fuzzy numbers simultaneously like Chen \& Chen (2009) and Bakar \& Gegov $(2014 ; 2015)$ while pairwise ranking is the capability of a method to ranking only two fuzzy numbers at one time such as Bakar et al. $(2010 ; 2012)$ and Dat et al. (2012). Although, there are different capabilities in terms of ranking fuzzy numbers, both kinds of ranking fuzzy numbers methods follow the same basic algorithms when ranking fuzzy numbers. In accordance to aforementioned ranking methods, basic algorithms for ranking fuzzy numbers are signified as Algorithm 1 and are shown as follows.

\section{Algorithm 1}

1) Assign value towards each fuzzy numbers under consideration whereby this value is called as assignment.

2) Make comparison based on assignment obtained. This step is also known as sorting stage.

In this case, the number of assignments and comparisons computed depends on either method uses direct ranking or pairwise ranking. This is due to the fact that direct ranking can simultaneously ranks all fuzzy numbers under consideration while pairwise ranking needs more steps than the former before the fuzzy numbers under consideration are ranked. It is worth noticing here that the comparisons step mentioned above is a sorting - based step. In Table 6, comparison between direct ranking and pairwise ranking in terms of number of assignments and comparison are illustrated.

Table 6: Comparison between direct ranking and pairwise ranking

\begin{tabular}{ccccc}
\hline $\begin{array}{c}\text { No. of } \\
\text { Fuzzy } \\
\text { Numbers }\end{array}$ & \multicolumn{2}{c}{ Direct Ranking } & \multicolumn{2}{c}{ Pairwise Ranking } \\
\cline { 2 - 5 } & $\begin{array}{c}\text { No. of } \\
\text { Assignments }\end{array}$ & $\begin{array}{c}\text { No. of } \\
\text { Comparisons }\end{array}$ & $\begin{array}{c}\text { No. of } \\
\text { Assignments }\end{array}$ & $\begin{array}{c}\text { No. of } \\
\text { Comparisons }\end{array}$ \\
\hline 2 & 2 & 1 & 2 & 1 \\
3 & 3 & 1 & 6 & 3 \\
4 & 4 & 1 & 12 & 6 \\
5 & 5 & 1 & 20 & 10 \\
6 & 6 & 1 & 30 & 15 \\
7 & 7 & 1 & 42 & 21 \\
8 & 8 & 1 & 56 & 28 \\
\hline
\end{tabular}

Although, Table 6 shows that direct ranking and pairwise ranking are different in terms of capability in ranking fuzzy numbers, both sometimes need additional operations to ranking fuzzy numbers appropriately. This is because in certain situation, a method is incapable to ranking fuzzy numbers properly only if one approach is used. For example, ranking method by Yu et al. (2013) ranks each fuzzy number under consideration using centroid approach. If the method produces similar ranking order for different fuzzy numbers, then the epsilon degree approach is next utilised to finalise the ranking result obtained. This points out that Yu et al. (2013) ranking method needs an additional operation which is epsilon degree approach, to ranking fuzzy numbers with correct ranking order such that the ranking result is consistent with human intuition. Same situation is faced by Cheng (1998), Chen \& Lu (2002) and Kumar et al. (2010) ranking methods where additional operations are needed when they want to appropriately rank the fuzzy 
numbers. Thus, in some cases, incorporation of additional operation is compulsary when correct ordering for fuzzy numbers is targeted to achieve. Based on this verification, Algorithm 1 mentioned earlier is extended to Algorithm 2, whereby additional algorithms are included as a result of additional operation used to ranking fuzzy numbers. In this respect, Algorithm 2 is illustrated as follows.

\section{Algorithm 2}

1) Assign value towards each fuzzy numbers under consideration whereby this value is called initial assignment.

2) Make comparison based on assignment obtain which is defined as initial comparison.

3) Assign value towards each fuzzy numbers under consideration for second time whereby this value is called secondary assignment.

4) Make comparison based on secondary assignment obtain which is defined as secondary comparison.

It has to be noted that steps 1 and 2 of Algorithm 1 are changed to initial assignment and initial comparison in Algorithm 2 respectively since it involves additional assignment and comparison which are in steps 3 and 4 respectively. Therefore, regardless a method uses direct ranking or pairwise ranking, incorporation of additional operation leads to utilisation of secondary assignment and secondary comparison in ranking fuzzy numbers algorithm. Table 5 presents comparison between direct ranking, direct ranking with additional operation, pairwise ranking and pairwise ranking with additional operation.

Table 7: Comparison between direct ranking and pairwise ranking.

\begin{tabular}{ccccc}
\hline \multirow{2}{*}{ Algorithm } & \multicolumn{2}{c}{ Direct Ranking } & \multicolumn{2}{c}{ Pairwise Ranking } \\
\cline { 2 - 5 } & $\begin{array}{c}\text { Without Additional } \\
\text { Operation }\end{array}$ & $\begin{array}{c}\text { With Additional } \\
\text { Operation }\end{array}$ & $\begin{array}{c}\text { Without Additional } \\
\text { Operation }\end{array}$ & $\begin{array}{c}\text { With Additional } \\
\text { operation }\end{array}$ \\
\hline Initial Assignment & Yes & Yes & Yes & Yes \\
Initial Comparison & Yes & Yes & Yes & Yes \\
Secondary Assignment & No & Yes & No & Yes \\
Secondary Comparison & No & Yes & No & Yes \\
\hline
\end{tabular}

Based on discussion made above, evaluation of efficiency in terms of ranking algorithm is classified into four namely very efficient, slightly efficient, slightly inefficient and very inefficient. All of these classes are determined through examining capability of a method in ranking more than two fuzzy numbers simultaneously. Based on Table 6 and Table 7, Table 8 and Figure 13 are constructed.

Table 8: Evaluation of Efficiency

\begin{tabular}{|c|c|c|c|c|}
\hline \multirow{3}{*}{$\begin{array}{c}\text { No. of Fuzzy } \\
\text { numbers }\end{array}$} & \multicolumn{4}{|c|}{ Efficiency } \\
\hline & \multicolumn{2}{|c|}{ Direct Ranking } & \multicolumn{2}{|c|}{ Pairwise Ranking } \\
\hline & $\begin{array}{l}\text { Without Additional } \\
\text { Computation }\end{array}$ & $\begin{array}{l}\text { With Additional } \\
\text { Computation }\end{array}$ & $\begin{array}{c}\text { Without Additional } \\
\text { Computation }\end{array}$ & $\begin{array}{l}\text { With Additional } \\
\text { Computation }\end{array}$ \\
\hline 2 & $2_{I A}$ & $2_{I A}$ & $2_{I A}$ & $2_{I A}$ \\
\hline 3 & $3_{I A}$ & $3_{I A}+3_{I C}+3_{S A}+3_{S C}$ & $6_{I A}+3_{I C}$ & $6_{I A}+3_{I C}+6_{S A}+3_{S C}$ \\
\hline 4 & $4_{I A}$ & $4_{I A}+4_{I C}+4_{S A}+4_{S C}$ & $12_{I A}+6_{I C}$ & $12_{I A}+6_{I C}+12_{S A}+6_{S C}$ \\
\hline 5 & $5_{I A}$ & $5_{I A}+5_{I C}+5_{S A}+5_{S C}$ & $20_{I A}+10_{I C}$ & $20_{I A}+10_{I C}+20_{S A}+10_{S C}$ \\
\hline 6 & $6_{I A}$ & $6_{I A}+6_{I C}+6_{S A}+6_{S C}$ & $30_{I A}+15_{I C}$ & $30_{I A}+15_{I C}+30_{S A}+15_{S C}$ \\
\hline 7 & $7_{I A}$ & $7_{I A}+7_{I C}+7_{S A}+7_{S C}$ & $42_{I A}+21_{I C}$ & $42_{I A}+21_{I C}+42_{S A}+21_{S C}$ \\
\hline 8 & $8_{I A}$ & $8_{I A}+8_{I C}+8_{S A}+8_{S C}$ & $56_{I A}+28_{I C}$ & $56_{I A}+28_{I C}+56_{S A}+28_{S C}$ \\
\hline$N$ & $f(N)=N$ & $f(N)=4 N$ & $f(N)=\frac{3}{2} N^{2}-\frac{3}{2} N$ & $f(N)=3 N^{2}-3 N$ \\
\hline $\begin{array}{l}\text { Efficiency } \\
\text { Classification }\end{array}$ & Very Efficient & Slightly Efficient & Slightly Inefficient & Very Inefficient \\
\hline
\end{tabular}


No. of operation

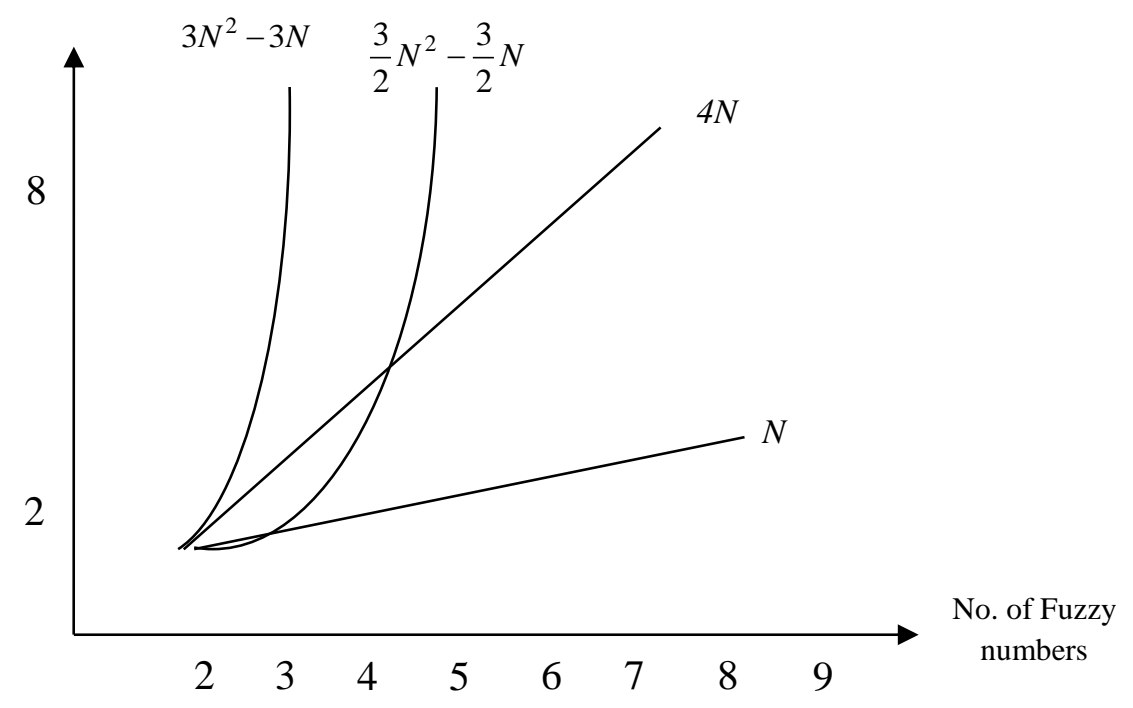

Figure 13: Evaluation of efficiency

Table 9 presents efficiency evaluations comparisons on ranking fuzzy numbers among established ranking methods.

Table 9: Efficiency Comparison among Ranking Methods

\begin{tabular}{lc}
\hline \multicolumn{1}{c}{ Ranking Method } & Level of Efficiency \\
\hline $1-$ Cheng (1998) & Slightly Inefficient \\
\hline $2-$ Wu \& Mendel (2009) & Slightly Efficient \\
\hline $1-$ Kumar et al. (2010) & Slightly Inefficient \\
\hline $1-$ Dat et al. (2012) & Slightly Inefficient \\
\hline $1-$ Yu et al. (2013) for $\alpha=0$ & Slightly Efficient \\
\hline $1-$ Yu et al. (2013) for $\alpha=0.5$ & Slightly Efficient \\
\hline $1-$ Yu et al. (2013) for $\alpha=1$ & Slightly Efficient \\
\hline $1-$ Zhang et al. (2014) for $\alpha=0$ & Very Inefficient \\
\hline $1-$ Zhang et al. (2014) for $\alpha=0.5$ & Very Inefficient \\
\hline $1-$ Zhang et al. (2014) for $\alpha=1$ & Very Inefficient \\
\hline $1-$ Bakar \& Gegov (2014) & Very Efficient \\
\hline Z - Bakar \& Gegov (2015) & Very Efficient \\
\hline
\end{tabular}

' 1 ' denotes that the ranking method is developed for ranking type - 1 fuzzy numbers ' 2 ' denotes that the ranking method is developed for ranking type -2 fuzzy numbers

' $\mathrm{Z}$ ' denotes that the ranking method is developed for ranking $\mathrm{Z}$ - number.

It is clearly indicated in Table 9 and Figure 13, ranking methods such as $2-\mathrm{Wu} \&$ Mendel (2009), $1-\mathrm{Yu}$ et al. (2013) for all values of $\alpha, 1$ - Bakar \& Gegov (2014) and Z - Bakar \& Gegov (2015) which rank fuzzy numbers using direct ranking are represented by linear function while others remaining ranking methods which rank fuzzy numbers using pairwise ranking are characterised by quadratic function. This implies that ranking methods that rank fuzzy numbers using direct ranking is more efficient than ranking methods using pairwise ranking as the latter produce a quadratic function. It is also signified in the analysis where ranking methods by $1-$ Bakar \& Gegov 
(2014) and Z - Bakar \& Gegov (2015) are four times (4 times) more efficient than ranking methods that use direct ranking with additional operation like $2-\mathrm{Wu} \&$ Mendel (2009), 1 - Yu et al. (2013) for all values of $\alpha$ as functions for efficiency classification of direct ranking without additional operation and direct ranking with additional operation are $f(N)=N$ and $f(N)=4 N$ respectively. For pairwise ranking, method with additional operation like Zhang et al. (2014) for all values of $\alpha$, is twice less efficient than one without additional operation ( 1 - Cheng, 1998; 1 - Dat et al., 2012) where functions for efficiency classification obtained are $f(N)=3 N^{2}-3 N$ and $f(N)=\frac{3}{2} N^{2}-\frac{3}{2} N$ for method with additional operation and method without additional operation respectively.

Therefore, based on these descriptions, ranking methods use direct ranking without additional computation is classified as very efficient, ranking methods use direct ranking with additional operation is considered as slightly efficient, ranking methods use pairwise ranking without additional operation is classified as slightly inefficient and ranking methods use pairwise ranking with additional operation is considered as very inefficient.

\section{Conclusion}

This paper presents a detailed survey on validation techniques for ranking fuzzy numbers that are associated with properties of fuzzy quantities as well as consistency and efficiency of ranking operations. Although there is plenty of information available with regard to ranking type -1 and type -2 fuzzy numbers, there is still lack of information on ranking $\mathrm{Z}$ - numbers. Therefore, for future research on ranking fuzzy numbers, further investigations for validation of $\mathrm{Z}$ - numbers should be carried out. In conclusion, the validation techniques presented in this paper would be quite useful for evaluating the quality, consistency and efficiency of the ranking method used.

\section{References.}

[1] Chen, S. M., Munif, A., Chen, G-S., Liu, H - S, \&Kuo, B - C. (2012). Fuzzy Risk Analysis Based on Ranking Generalized Fuzzy Numbers with Different Heights and Different Spreads. Expert Systems with Applications 39, pp. $6320-6334$.

[2] Wu, D., Wu, D. D., Zhang, Y., \& Olson, D. L. (2013). Supply chain outsourcing risk using integrated stochastic - fuzzy optimization approach. Information Sciences 235, pp. $242-258$.

[3] Bermudez, J. D., Segura, J. V., \&Vercher, E. (2012).A multi - objective genetic algorithm for cardinality constrained fuzzy portfolio selection. Fuzzy Sets and Systems 188 (1), pp. $16-26$.

[4] Ebrahimnejad, S., Mousavi, S. M., Moghaddam, R. T., Hashemi, H., \&Vahdani, B. (2012).A novel two - phase group decision making approach for construction project selection in a fuzzy environment.Applied Mathematical Modelling 36 (9), pp. 4197 - 4217.

[5] Morais, D. C., \& Almeida, A. T. (2012). Group decision making on water resources based on analysis of individual rankings. Omega 40 (1), pp. $42-52$.

[6] Deng, H. (2013). Comparing and ranking fuzzy numbers using ideal solutions.Applied Mathematical Modelling, in press.

[7] Zimmermann, H - J. (2000). An application - oriented view of modelling uncertainty.European Journal of Operational Research122, pp. $190-198$.

[8] Kumar, A., Singh, P., Kaur P. and Kaur A.(2010). A New Approach for Ranking Generalized Trapezoidal Fuzzy Numbers, World Academy of Science, Engineering and Technology 68,pp. 229 - 302.

[9] Jain, R. (1976). Decision-Making In The Presence of Fuzzy Variable.IEEE Transactions on Man and Cybernetic 6, pp. $698-703$.

[10] Chen, S. M., \& Chen, J. H. (2009). Fuzzy Risk Analysis Based on Ranking Generalized Fuzzy Numbers with Different Heights and Different Spreads. Expert Systems with Applications 36, pp. 6833 - 6842.

[11] Bakar, A. S. A., Mohamad, D., \&Sulaiman, N. H. (2010). Ranking Fuzzy Numbers using Similarity Measure with Centroid. IEEE International Conference on Science and Social Research, pp. 58 - 63.

[12] Chen, S.M. \&Sanguansat, K. (2011). Analyzing fuzzy risk based on a new fuzzy ranking method between generalized fuzzy numbers. Expert System with Applications 38, pp. 2163 - 2171. 
[13] Bakar, A. S. A., Mohamad, D., \& Sulaiman, N. H. (2012). Distance - Based Ranking Fuzzy Numbers. Advances in Computational Mathematics and Its Applications 1(3), pp. 146 - 150.

[14] Dat, L. Q., Yu, V. F., \& Chou, S. Y. (2012). An Improved Ranking Method for Fuzzy Numbers Based on the Centroid Index, International Journal of Fuzzy Systems 14 (3), pp. 413 - 419.

[15] Yu, V. F., Chi, H. T. X., \& Shen, C. W. (2013). Ranking Fuzzy Numbers based on Epsilon - Deviation Degree, Applied Soft Computing 13 (8), pp. 3621 - 3627.

[16] Bakar, A. S. A. \&Gegov, A. (2014). Ranking of fuzzy numbers based centroid point and spread, Journal of Intelligent and Fuzzy Systems 27, pp. 1179 - 1186.

[17] Kang, B., Wei, D., Li, Y., \& Deng, Y. (2012a). A method of converting Z-numbers to classical fuzzy numbers, Journal of Information and Computational Science9 (3), pp. $703-709$.

[18] Zadeh, L. A. (2011).A note on Z - numbers, Information Sciences 181, pp. 2923 - 2932.

[19] Yager, R. R. (2012a). On A View of Zadeh's Z - Numbers.Springer-Verlag Berlin Heidelberg, pp. 90 - 101.

[20] Yager, R. R. (2012b). On Z - Valuations using Zadeh's Z - Numbers. International Journal of Intelligent Systems 27 (3), pp. $259-278$.

[21] Kang, B., Wei, D., Li, Y., \& Deng, Y. (2012b). Decision Making using Z - Numbers Under Uncertain Environemnt. Journal of Computational Information Systems 8 (7), pp. 2807 - 2814.

[22] Gardashova, L. A. (2014). Application of Operational Approaches to Solving Decision Making Problem Using Z - Numbers. Journal of Applied Mathematics 5 (9), pp. 1323 - 1334.

[23] Shieh, B.S. (2007). An Approach to Centroids of Fuzzy Numbers .International Journal of Fuzzy Systems 9 , pp. $51-54$.

[24] Cheng, C. H. (1998). A New Approach for Ranking Fuzzy Numbers by Distance Method.Fuzzy Sets and System 95, pp. $307-317$.

[25] Chu, C.T. \&Tsao, C.T. (2002). Ranking Fuzzy Numbers with an Area Between the Centroid Point and Original Point. Computer and Mathematics with Applications 43, pp. 111 - 117.

[26] Allahviranloo, T. \&Saneifard, R. (2012). Defuzzification Method for Ranking Fuzzy Numbers based on Center of Gravity. Iranian Journal of Fuzzy Systems 9 (6), pp. $57-67$.

[27] Abbasbandy, S. \& Asady, B. (2006). Ranking of Fuzzy Numbers by Sign Distance. Iranian Information Science 176, pp. $2405-2416$.

[28] Abbasbandy, S. \& Hajjari, T. (2009). A New Approach for Ranking of Trapezoidal Fuzzy Numbers. Computer and Mathematics with Applications 57, pp. $413-419$.

[29] Wang, Z.X., Liu, Y.J., Fan, Z.P. \& Feng, B. (2009). Ranking L-R Fuzzy Numbers based on Deviation Degree. Information Scicences 179, pp. 2070 - 2077.

[30] Hadi, A.M. \& Allame, M. (2010). On The Relation Between A Fuzzy Numbers and Its Centroid. Computer and Mathematics with Applications 59, pp. 3578 - 3582.

[31] Wang, Y.M., Chin, K.S., Poon, G.K.K. \& Yang, J.B. (2009). Risk Evaluation in Failure Mode and Effects Using Fuzzy Weighted Geometric Mean. Expert Systems with Applications 36, pp. 1195 - 1207.

[32] Salahshour, S., Abbasbandy, S. \& Allahviranloo, T. (2011). Ranking Fuzzy Numbers Using Fuzzy Maximum and Minimum Points. EUSFLAT - LFA, Aix-les-Bains, France, pp. $763-769$.

[33] Bakar, A. S. A. \& Gegov, A. (2015). Multi-Layer Decision Methodology for Ranking Z - Numbers, International Journal of Computational Intelligent Systems 8 (2), pp. 395 - 406.

[34] Brunelli, M. \& Mezei, J. (2013). How Different Are Ranking Methods for Fuzzy Numbers? A Numerical Study, International Journal of Approximate Reasoning 54, pp. 627 - 639.

[35] Elamvazuthi, I., Ganesan, T., Vasant, P., \& Webb, J.F. (2009). Application of A Fuzzy Programming Technique to Production Planning in the Textile Industry, International Journal of Computer Science and Information Security 6(3), pp. 238 - 243.

[36] Mousavi, S.M., Mirdamadi, S., Siadat, A., Dantan, J-Y \& Moghadam, R.T. (2015). An Intuitionistic Fuzzy Grey Model for Selection Problems With Application to the Inspection in Manufacturing Firms, Engineering Applications of Artificial Intelligence 39, pp. 157 - 167.

[37] Sui, S., Li, Y. \& Tong, S. (2015). Adaptive Fuzzy Control Design and Applications of Uncertain Stochastic Nonlinear Systems With Input Saturation. Neurocomputing 156, pp. $42-51$.

[38] Liem, D.T., Truong, D.Q. \& Ahn, K.K. (2015). A Torque Estimator Using Online Turning Grey Fuzzy PID for Applications to Torque-Sensorless Control of DC Motors, Mechatronics 26, pp. 45 - 63.

[39] Collan, M. (2009). New Method for Real Option Valuation using Fuzzy Numbers. IAMSR Research Report, Turku, Institute for Advanced Management Systems Research. 